\title{
Design of a Superconducting Magnet System for the AEGIS Experiment at CERN
}

\author{
Alexey Dudarev, Michael Doser, Diego Perini, and Herman ten Kate
}

\begin{abstract}
The new AEGIS (Antimatter Experiment: Gravity, Interferometry, Spectroscopy) Experiment will be installed in the Antiproton Decelerator hall at CERN. The main goal is to measure the Earth's gravitational acceleration of antihydrogen atoms. The experiment consists of two high-homogeneity solenoids placed on the same axis. The $5 \mathrm{~T}$ magnet is part of a cylindrical Penning trap to catch and to accumulate antiprotons delivered by the decelerator. The antihydrogen is then produced in the $1 \mathrm{~T}$ region where sub-kelvin antiproton temperatures provided by the dilution refrigerator are required to form a slowly-moving beam of antihydrogen. The helium bath cooled superconducting magnets; the different traps and the dilution refrigerator are integrated in a common cryostat with an internal vacuum barrier between the insulating cryogenic vacuum and the very high beam vacuum. In addition, the magnet system has to guarantee a smooth transition between the $5 \mathrm{~T}$ and the $1 \mathrm{~T}$ magnetic field areas required for a loss-free transfer of antiprotons and positrons from the trapping region to the antihydrogen production area. In this paper the design of this AEGIS magnet system is presented and discussed.
\end{abstract}

Index Terms-Anti-hydrogen, cryostat, gravitational acceleration, superconducting magnet.

\section{INTRODUCTION}

$\mathbf{T}$ HERE is a number of theoretical arguments to believe that the gravitational interaction of matter and antimatter should be identical. However, same quantum gravity models leave room for possible differences in the behavior of matter and antimatter in the Earth's gravitational field. These supposed differences would violate the weak equivalence principle for antimatter. So far there has not been a single direct measurement of gravity on antimatter and all available information has been extrapolated from matter results or infers from indirect measurements that often are model dependent.

The AEGIS Experiment [1]-[3] is currently under construction in the Antiproton Decelerator (AD) hall at CERN. It aims to directly measure the gravitational acceleration by detecting the vertical deflection of the antihydrogen beam, after a flight path of about 1 meter, with a $1 \%$ relative precision. The essential steps leading to the production of antihydrogen and the measurement of its gravitational interaction are: production of positrons from a Surko-type source and accumulator; capture

Manuscript received August 01, 2010; accepted September 03, 2010. Date of publication January 24, 2011; date of current version May 27, 2011. This project was supported by the AEGIS Collaboration, presently about 50 scientific authors and supported in part by CERN.

The authors are with CERN, the European Center for Nuclear Research, POB Geneva 23, 1211 Geneva, Switzerland (e-mail: Alexey.Dudarev@cern.ch).

Digital Object Identifier 10.1109/TASC.2010.2100345

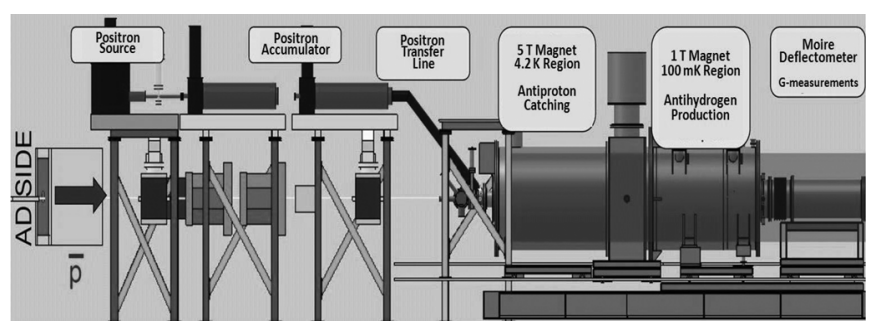

Fig. 1. The AEGIS experiment: production of positrons from a source and accumulator; capture and accumulation of antiprotons from the $\mathrm{AD}$ at $4.2 \mathrm{~K} \&$ $5 \mathrm{~T}$; production of positronium by bombardment of a nanoporous material with positrons at $0.1 \mathrm{~K} \& 1 \mathrm{~T}$; recombination of antihydrogen; formation of an antihydrogen beam; determination of gravity in a Moiré deflectometer.

and accumulation of antiprotons from the Antiproton Decelerator in a cylindrical Penning trap; cooling of the antiprotons to sub-kelvin temperature; production of positronium by bombardment of a cryogenic nanoporous material with an intense positron pulse; excitation of the positronium to a Rydberg state; pulsed formation of antihydrogen by resonant charge exchange; pulsed formation of an antihydrogen beam by Stark acceleration with inhomogeneous electric fields; determination of Earth's gravitational acceleration in a two-grating Moiré deflectometer coupled to a position-sensitive detector. The location of different sub-systems is shown in Fig. 1.

To guarantee proper catching of particles the AEGIS experiment requires a solenoidal magnetic field of a special configuration. Due to the high energy of the antiproton beam from the decelerator the first stage of trapping has to be realized in the 5 T homogeneous magnetic fields. Next the "cooled" antiprotons arrive in the $1 \mathrm{~T}$ region for the further cooling and their recombination with the positronium.

This paper is focused on the design of the AEGIS superconducting magnet system with respect to geometry of coils, their protection, powering and cryostating. The magnetic field adjustment and mapping are discussed as well.

\section{REQUIREMENTS}

Serving as parts of two Penning traps the AEGIS solenoids have to provide a magnetic field of rather high homogeneity. The requirements on the field homogeneity in both magnets are similar. It must be better than $10^{-3}$ in a cylinder of diameter of $100 \mathrm{~mm}$ and $600 \mathrm{~mm}$ in length. Inside this space there has to be a region $\left(\varnothing 10\right.$ by $\left.100 \mathrm{~mm}^{2}\right)$ with homogeneity about $10^{-5}$.

The solenoids have to be placed in separated cryostats with 150-200 mm gap to allow the installation of different electrical traps and detectors. The transition of the magnetic field from the $5 \mathrm{~T}$ to the $1 \mathrm{~T}$ region has to be smooth. An additional coil (so called anticoil) to reduce the axial gradient of magnetic field in 


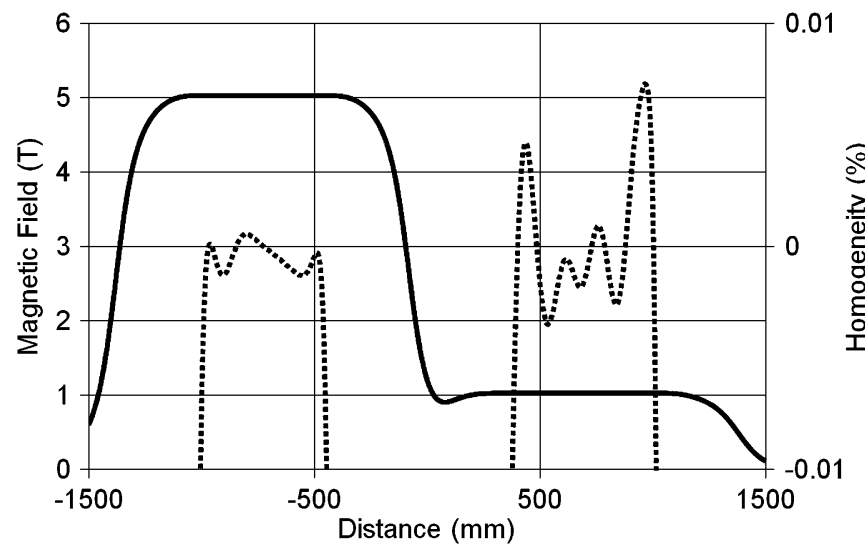

Fig. 2. AEGIS requires two high homogeneity areas of $5 \mathrm{~T}$ and $1 \mathrm{~T}$ with a smooth transition in between. The calculated magnetic field distribution (solid line) and its homogeneity (dotted line) are meeting the requirements with some margin.

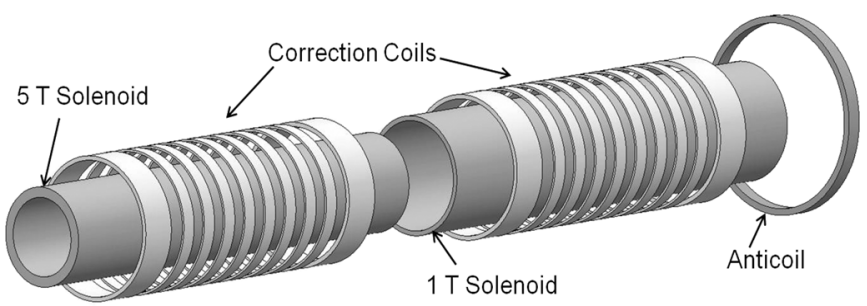

Fig. 3. The AEGIS magnet system consists of 23 superconducting coils. Two big correction coils for each solenoid provide rough steering of the field and eight small correction coils are making fine tuning. The anticoil has an opposite current to minimize the field gradient in the deflectometer region.

the Moiré deflectometer area is required as well. To summarize the AEGIS requirements the calculated magnetic field distribution and homogeneity are shown in Fig. 2.

\section{LAYOUT OF COILS}

In an earlier stage of the project a geometrical layout with the coils charged in series was considered. It is possible to find a good geometrical solution but such a system is extremely sensitive to any variation of parameters and it makes impossible to use the two magnets independently or at different values of magnetic field. Therefore, a layout is chosen with a long solenoid creating a bias field surrounded by a set of concentric correction coils. There was even a proposal to make two identical magnets and cryostats. It would allow covering a wide range of magnetic field. This option was then canceled because of certain space requirements in the $1 \mathrm{~T}$ region. This caused a change of the diameters of the main solenoids but the layout of the set of correction coils did not change because their spools were already ordered. The final layout of the AEGIS magnet system is shown in Fig. 3. There are twenty three superconducting coils in total: a $5 \mathrm{~T}$ solenoid with 10 correction coils and a $1 \mathrm{~T}$ solenoid with the same amount of correction coils and the anticoil. All coils are placed on the same axis. The main parameters of the coils are given in Table I.

\section{A. $5 T$ and $1 T$ Solenoids}

The solenoids are wound onto a $1.3 \mathrm{~m}$ long bobbin of fiberglass reinforced epoxy, which is carefully machined to
TABLE I

MAIN PARAMETERS OF AEgIS COILS

\begin{tabular}{ccccc}
\hline \hline & $\begin{array}{c}\mathbf{5} \text { T } \\
\text { Solenoid }\end{array}$ & $\begin{array}{c}\mathbf{1 ~ T} \\
\text { Solenoid }\end{array}$ & $\begin{array}{c}\text { Big / Small } \\
\text { Correction } \\
\text { Coil }\end{array}$ & Anticoil \\
\hline $\begin{array}{c}\text { Number of coils } \\
\quad \text { Conductor }\end{array}$ & 1 & 1 & $4 / 16$ & 1 \\
$\begin{array}{c}\text { dimensions (mm) } \\
\text { Critical current } \\
\text { at 5 T (A) }\end{array}$ & $1.25 \times 0.8$ & $1.25 \times 0.8$ & $\varnothing 0.64$ & $\varnothing 0.64$ \\
$\begin{array}{c}\text { Cu /NbTi ratio } \\
\text { RRR }\end{array}$ & 4.2 & 4.2 & 3.7 & 3.7 \\
$\begin{array}{c}\text { Inner diameter } \\
\text { (mm) }\end{array}$ & 180 & 250 & $310 / 320$ & 420 \\
$\begin{array}{c}\text { Outer diameter } \\
\text { (mm) }\end{array}$ & 228 & 274 & 330 & 450 \\
$\begin{array}{c}\text { Coil length (mm) } \\
\begin{array}{c}\text { Operating current } \\
\text { (A) }\end{array}\end{array}$ & 1300 & 1300 & $100 / 40$ & 40 \\
\begin{tabular}{c} 
Inductance (H) \\
\hline \hline
\end{tabular} & 194 & 85 & up to $10 / 5$ & 17 \\
\hline
\end{tabular}

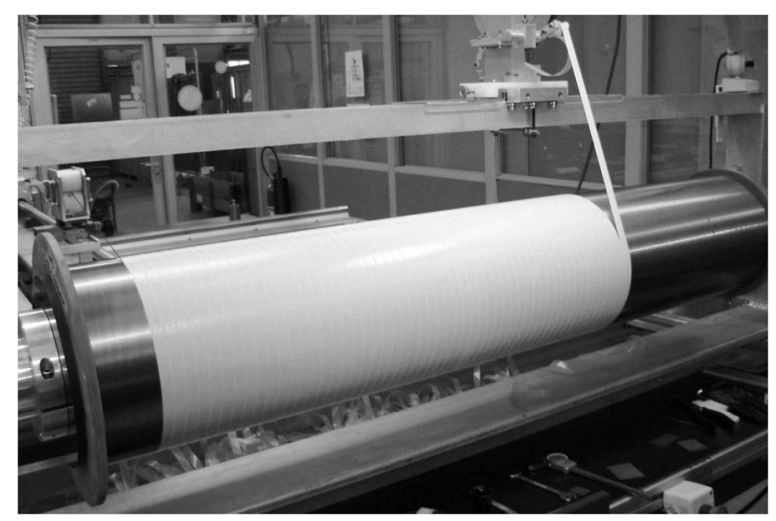

Fig. 4. Winding of the $1 \mathrm{~T}$ solenoid: to place properly the insulation between layers the fiberglass tape is accurately wound together with the first turns of the conductor layer.

obtain the azimuthal symmetry and the field homogeneity. For the same reason of symmetry, even number of turns is chosen to avoid any influence of the current return loop. Also the routing of the superconducting bus bars is organized in a bifilar way. One unit length of conductor is used for winding of both solenoids to minimize irregularities in magnetic field. The coils are wound with enamel-insulated rectangular $\mathrm{NbTi}$ wire with dimension of 1.25 by $0.8 \mathrm{~mm}^{2}$.

The critical current of the conductor is more than twice the operating current at the peak magnetic field. There is an interlayer insulation tape of $0.2 \mathrm{~mm}$ thickness, which is wound over the already finished conductor layer as shown in Fig. 4. The glass tape has a high content of an epoxy compound that allows performing the impregnation while on the winding machine by heating of the finished coils to 120 degrees.

The production of solenoids has started in June 2010 after setting up the winding machine and winding a short model coil. 


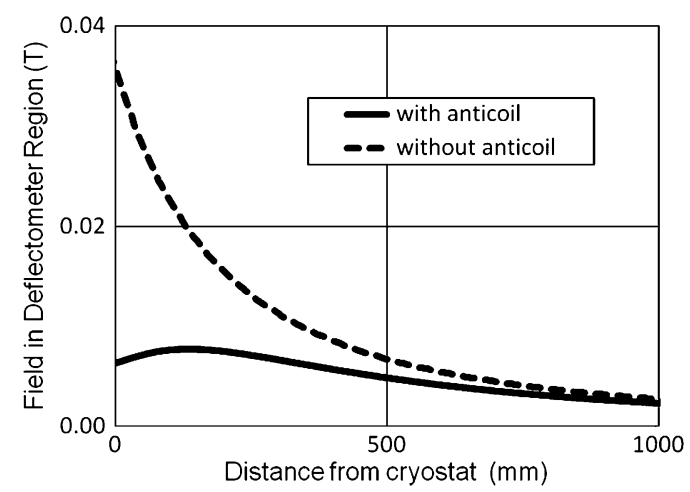

Fig. 5. The additional anticoil is place at the extremity of the $1 \mathrm{~T}$ magnet to minimize the influence of the magnetic field on the antihydrogen beam in the gravity measurement zone of the Moiré deflectometer. The field gradient is reduced by a few times.

The model coil was carefully checked and measured. It is electrically sound and passed a thermal shock test in a liquid nitrogen bath without any observable damage.

\section{B. Correction Coils and Anticoil}

The number of correction coils and their exact location may be chosen rather arbitrarily if coils are charged independently. There are two types of correction coils. Big coils are place outside the requested homogeneous area and they provide a rough correction of the magnetic field. Small coils make the fine tuning to guarantee the high field homogeneity. All coils are wound in grooves machined in a thick fiberglass cylinder, concentric with the main coils and fitting precisely in between the flanges of the main solenoids.

As mentioned above, both $5 \mathrm{~T}$ and $1 \mathrm{~T}$ solenoids have identical layout of correction coils (see Fig. 3). The anticoil is fixed to the $1 \mathrm{~T}$ correction coils cylinder and it is charged with opposite current to minimize and equalize the gradient of the magnetic field in the deflectometer area as shown in Fig. 5.

The correction coils and the anticoil do not have high operating currents and their stored energy is not significant. The 0.64 $\mathrm{mm}$ diameter $\mathrm{NbTi}$ wire is used for their windings. The insulation between layers is present like in the main solenoids. There is no significant field gradient on the correction coils so the mechanical forces are negligible. The maximum attraction force between solenoids is about $2 \mathrm{kN}$. The repelling force seen by the anticoil is $1.5 \mathrm{kN}$.

\section{CRyostat}

The Antiproton Decelerator hall is not equipped with a cryogenic plant. Consequently, all experiments in this area are using cryogenic liquids transported from other facilities at CERN. For this reason it is decided to maximize the volumes of liquid helium and nitrogen stored inside the cryostats to enable a few days of continuous operation without refilling. The cryostat comprises three main parts: $1 \mathrm{~T}$ and $5 \mathrm{~T}$ cryostats for the magnets and a central insertion region (see Fig. 6).

Regarding the requirements of the superconducting magnets the cryostat is designed in a classical way. Each magnet is placed on the central axis of an independent helium vessel of about 800 liter. Superconducting bus bars are following a routing down to

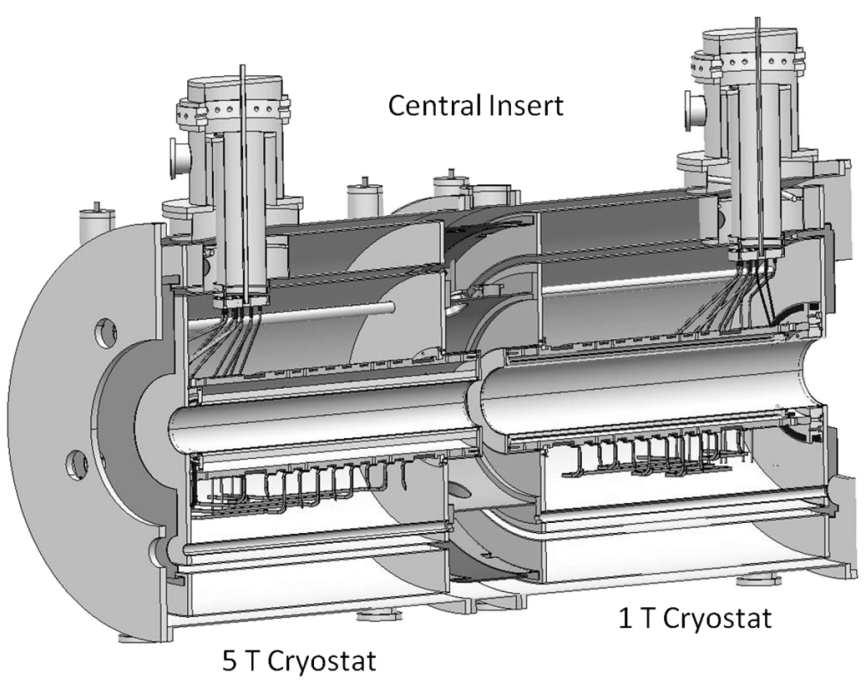

Fig. 6. The AEGIS Experiment can be split in three independent parts to provide easy access to various internal traps and detectors (not shown), fixed to the central region and placed inside the $4.5 \mathrm{~K}$ bore tubes of the helium vessels of the magnets.

the bottom of the vessel and then up to a chimney equipped with current leads that makes the operation of the magnets almost independent from the liquid helium level. There is a 600 liter liquid nitrogen tank covered with blankets of multilayer super insulation.

A complexity is arising from the following requirement. The different traps, detectors and deflectometer must be placed in ultra high vacuum of $10^{-12}$ mbar that is much higher than isolation vacuum of the cryostat. It assumes the presence of an internal $4.5 \mathrm{~K}$ vacuum barrier that has to be mechanically detachable from the helium vessels when opening the experiment. The barrier must provide good thermalisation of different high voltage and measuring cables. It is decided to cool it by liquid helium coming from a dilution refrigerator cryostat which is located on top of the central insert. This cryostat shares the isolation and high vacuum with the experiment. There are other features such as cold optical windows for lasers, a special vessel around the antihydrogen detector which operates at $100 \mathrm{~K}$ in the $1 \mathrm{~T}$ region. The Moiré deflectometer has to be rigidly connected to the helium vessel of the $1 \mathrm{~T}$ magnet. At the antiproton decelerator side, there is a set of bellows making direct thermal link to room temperature.

At this moment it is impossible to make a good estimate of the total liquid helium consumption as many parts are yet under design. The heat load of the magnet system itself is expected to be about $4 \mathrm{~W}$ at operation conditions.

\section{Powering AND PROtection}

The sum of operating currents of all coils does not exceed $350 \mathrm{~A}$. The heat load caused by current leads is not a dominating factor in the total helium consumption. So it is decided to run the system by power supplies and not to use a persistent switch mode of operation.

Time stability of commercially available power supplies is sufficient to guarantee the requested magnetic field homogeneity. The correction coils operate at currents up to ten 


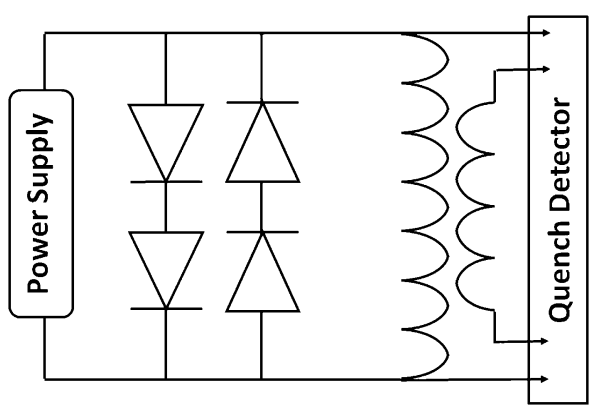

Fig. 7. Each coil is powered separately by its power supply. Room temperature protection diodes provide energy dissipation in the case of a quench or problems with power supplies. For the $5 \mathrm{~T}$ and $1 \mathrm{~T}$ solenoid circuits, the quench detection and switching off all power supplies is triggered by the differential voltage comparison between the coil and a pick-up coil of copper conductor wound on the external layer.

TABLE II

QUENCH SiMULATION RESULT

\begin{tabular}{ccc}
\hline \hline & 5 T Solenoid & 1 T Solenoid \\
\hline Hot spot temperature $(\mathrm{K})$ & 148 & 52 \\
Discharge time $(\mathrm{s})$ & 6 & 15 \\
Volume of quenched conductor $(\%)$ & 61 & 50 \\
Final coil resistance $(\Omega)$ & 26 & 3 \\
\hline \hline
\end{tabular}

amperes and require only $10^{-2}$ of current stability. For the power supplies of the main solenoids, this number has to be better than $10^{-3}$.

Fig. 7 shows the principal electrical circuit of high current coils. For the correction coils and the anticoil no quench detection system is needed. The various coils are equipped with a number of protection diodes to allow a reasonable ramp time of the current.

There are two layers of insulated copper wire wound on top of each coil. They serve both as a mechanical bandage and pick-up coil used for compensation of the coil voltage. The quench detection system is based on the differential comparison of the voltage across the superconducting coil to the voltage induced in the pick-up coil. There is no need for an active quench protection system. The main function of the quench detection system is switching off all power supplies to minimize warming up of the magnet system.

The quench protection analysis of the AEGIS solenoids is performed using the QLASA code developed by INFN. The summary of the simulation results is given in Table II.

Fig. 8 shows the hot spot temperature evolution during the internal dump of the total magnet stored energy into the solenoids under fully adiabatic conditions. The low values found indicate that no active protection system is required for the magnet system.

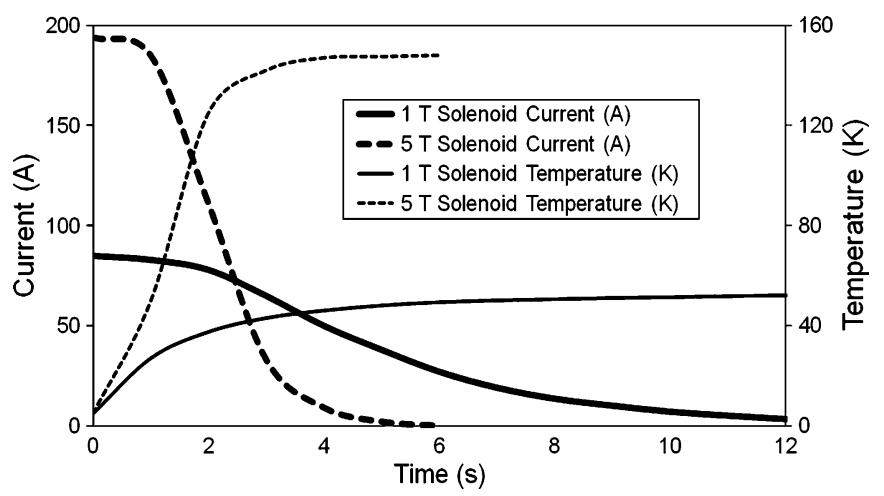

Fig. 8. Current decay and hot spot temperature versus time are calculated assuming all magnet stored energy is released adiabatically in the coils. The maximum temperature does not exceed $150 \mathrm{~K}$ in the $5 \mathrm{~T}$ magnet.

\section{TESTS}

The $5 \mathrm{~T}$ and $1 \mathrm{~T}$ solenoids with their correction coils will be tested in a vertical cryostat currently available at CERN. The test has to confirm the operational currents and to define the margins of the correction coils. Power supplies, current leads and the quench detection system will be checked at different combinations of currents in the coils.

The tuning of the entire magnet system has to be made after the cryostat integration. A room temperature bore tube will be mounted on the cryostat central axis. About twenty precisely calibrated 3-D Hall probes will provide continuous monitoring of the magnetic field. It will allow performing effectively the adjustment of correction currents. Finally the detailed magnetic field mapping will be done.

\section{CONCLUSION}

The manufacturing of the AEGIS magnet system has started and coil winding is in progress. The coils will be tested by the end of 2010 where after the system integration will start.

The design of the cryostats is in progress. The entire system will be operational in the middle of 2011.

\section{ACKNOWLEDGMENT}

The authors thank Pierre-Ange Giudici and Francois Garnier for the construction and setting up of the winding machine and development of a proper coil winding technique. We thank as well Gabriella Rolando for her effort to perform the simulated quench performance of the AEGIS magnets.

\section{REFERENCES}

[1] Proposal for the AEGIS Experiment at the CERN Antiproton Decelerator [Online]. Available: http://cdsweb.cern.ch/record/1037532/files/ spsc-2007-017.pdf

[2] A. Kellerbauer et al., "Proposed antimatter gravity measurement with an antihydrogen beam," Nucl. Instr. Meth. Phys. Res. B, vol. 266, pp. 351-356, 2008.

[3] D. Fabris et al., "The AEGIS detection system for gravity measurements," Nucl. Phys., A, vol. 834, pp. 751c-753c, 2010. 